\title{
Lucinactant attenuates pulmonary inflammatory response, preserves lung structure, and improves physiologic outcomes in a preterm lamb model of RDS
}

\author{
Marla R. Wolfson ${ }^{1-4}$, Jichuan Wu ${ }^{1,4}$, Terrence L. Hubert' ${ }^{1}$, Timothy J. Gregory ${ }^{5}$, Jan Mazela ${ }^{5,6}$ and Thomas H. Shaffer ${ }^{1,3,7}$
}

BACKGROUND: Acute inflammatory responses to supplemental oxygen and mechanical ventilation have been implicated in the pathophysiological sequelae of respiratory distress syndrome (RDS). Although surfactant replacement therapy (SRT) has contributed to lung stability, the effect on lung inflammation is inconclusive. Lucinactant contains sinapultide $\left(\mathrm{KL}_{4}\right)$, a novel synthetic peptide that functionally mimics surfactant protein B, a protein with anti-inflammatory properties. We tested the hypothesis that lucinactant may modulate lung inflammatory response to mechanical ventilation in the management of RDS and may confer greater protection than animal-derived surfactants.

METHODS: Preterm lambs (126.8 \pm 0.2 SD d gestation) were randomized to receive lucinactant, poractant alfa, beractant, or no surfactant and studied for $4 \mathrm{~h}$. Gas exchange and pulmonary function were assessed serially. Lung inflammation biomarkers and lung histology were assessed at termination.

RESULTS: SRT improved lung compliance relative to no SRT without significant difference between SRT groups. Lucinactant attenuated lung and systemic inflammatory response, supported oxygenation at lower ventilatory requirements, and preserved lung structural integrity to a greater degree than either no SRT or SRT with poractant alfa or beractant.

CONCLUSION: These data suggest that early intervention with lucinactant may more effectively mitigate pulmonary pathophysiological sequelae of RDS than the animal-derived surfactants poractant alfa or beractant.

M ajor risk factors for pulmonary pathophysiological sequelae from respiratory distress syndrome (RDS) in preterm infants include immature lung tissue, decreased host-defense and antioxidant systems, barotrauma, and volutrauma from mechanical ventilation, oxidant injury from supplemental oxygen exposure, and infection (1). A common thread among these risk factors is that they are known to cause an undesirable inflammatory response in thelung. High levels of certain inflammatory mediators correlate with other neonatal morbidities
(2). Theoretically, a reduction in the lung inflammatory load related to RDS may not only equate to an improvement in pulmonary outcome but also to a potential improvement in other neonatal morbidities such as necrotizing enterocolitis, retinopathy of prematurity, and cerebral palsy. Although more classical forms of anti-inflammatory agents such as early postnatal steroids have demonstrated acute improvements in oxygenation and pulmonary mechanics in preterm infants, because of significant adverse effects these agents are not considered safe for routine use in critically ill preterm infants (3). New therapies are urgently needed and being explored that specifically target the acute inflammatory responses in the lung (4-7).

Events leading to inflammation in the lung and subsequent remodeling of lung structure may begin as early as the immediate resuscitation period after birth, even when lung protective ventilatory strategies are used (8). Surfactant-treated ventilated preterm lambs and baboons with RDS develop bronchopulmonary dysplasia (BPD) associated with elevations of proinflammatory cytokines in the lungs $(9,10)$. These findings have also been reported in human infants who subsequently develop BPD $(11,12)$. These associations support the contention that inflammation in the lung is an important precursor to the development of BPD (1).

In addition to their role in reducing surface tension at the alveolar-capillary interface, surfactants have also been investigated for their role in modulating inflammation in the lung. Although a few studies have demonstrated that certain surfactants may increase inflammation $(13,14)$, other work has demonstrated that surfactant protein B (SP-B) protects the lung when inflammation is present (15). As an SP-B mimetic, the sinapultide $\left(\mathrm{KL}_{4}\right)$ peptide in lucinactant (Surfaxin, Discovery Laboratories, Warrington, PA), a synthetic surfactant recently approved by the US Food and Drug Administration, may have an inherent and distinct ability to modulate lung inflammation $(16,17)$. To test the hypothesis that lucinactant may modulate lung inflammatory response to mechanical ventilation in the

\footnotetext{
'Department of Physiology, Temple University School of Medicine, Philadelphia, Pennsylvania; ${ }^{2}$ Department of Medicine, Temple University School of Medicine, Philadelphia, Pennsylvania; ${ }^{3}$ Departments of Pediatrics, Temple University School of Medicine, Philadelphia, Pennsylvania; ${ }^{4}$ Center for Inflammation, Translational, and Clinical Lung Research, Temple University School of Medicine, Philadelphia, Pennsylvania; ${ }^{5}$ Discovery Laboratories, Inc., Warrington, Pennsylvania; ${ }^{6}$ Poznan University of Medical Sciences, Poznan, Poland; ${ }^{7}$ Center For Pediatric Research, Alfred I. duPont Hospital for Children, Wilmington, Delaware. Correspondence: Marla R. Wolfson (marla.wolfson@temple.edu)
} 
management of respiratory distress during early development and may confer greater protection against lung inflammation than animal-derived surfactants, we studied ventilated preterm lambs that were treated with lucinactant, poractant alfa (containing porcine SP-B and SP-C), or beractant (containing bovine SP-B and SP-C) via intratracheal bolus administration; a "no surfactant treatment" group served as a negative control.

\section{RESULTS}

The lambs averaged $126.8 \pm 0.20 \mathrm{SD} d$ gestation and $2.69 \pm 0.59$ SD $\mathrm{kg}$ body weight. There were no significant differences in age, gender, or weight between treatment groups.

\section{Physiological Profile}

Physiological responses are shown in Figure 1. There were no significant group differences at baseline for any parameter.
Initially, following surfactant replacement therapy (SRT), $\mathrm{PaO}_{2}$ (Figure 1a) increased $(P<0.001)$ as compared with both baseline values and negative controls, independent of SRT group. Over time, the oxygenation response in lambs treated with lucinactant was sustained and greater than in lambs treated with beractant $(P<0.001)$ or poractant alfa $(P<0.05)$ at $2-4 \mathrm{~h}$. By $4 \mathrm{~h}, \mathrm{PaO}_{2}$ in lambs treated with either beractant or poractant alfa was not significantly different than that in negative controls. $\mathrm{PaCO}_{2}$ (Figure $1 \mathrm{~b}$ ) decreased $(P<0.001)$ over time in all groups. Following SRT, there were small differences in $\mathrm{PaCO}_{2}$ across SRT groups over time. $\mathrm{PaCO}_{2}$ was lower than in negative controls for all SRT groups out to $2 \mathrm{~h}(P<0.001)$, not different between controls and lucinactant by $3 \mathrm{~h}$, and lower than in controls for beractant $(P<0.01)$ and poractant alfa $(P<0.05)$ at 3 and $4 \mathrm{~h}$, respectively. $\mathrm{pH}$ (Figure $1 \mathrm{c})$ increased $(P<0.001)$ over time in all SRT groups and was greater than in negative controls, a

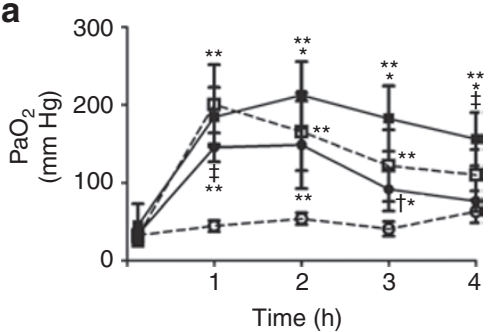

b
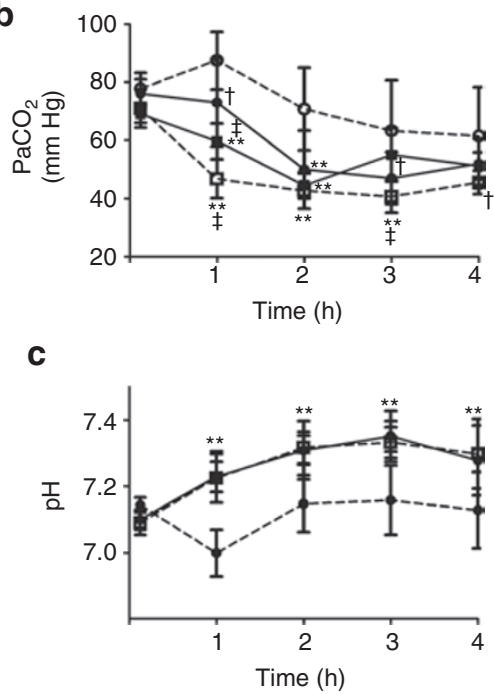

d

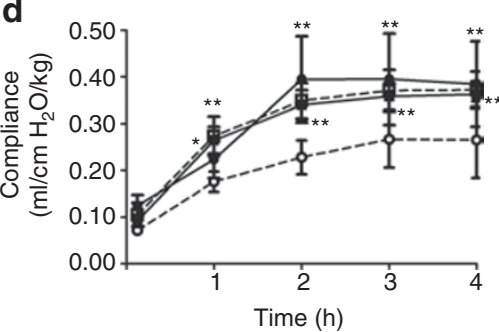

e

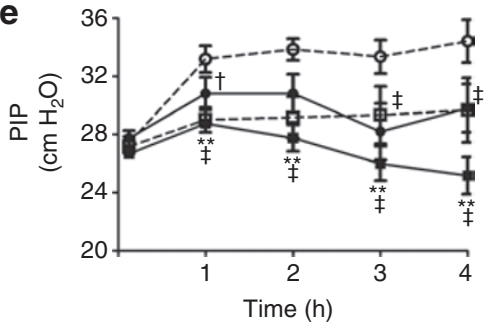

f



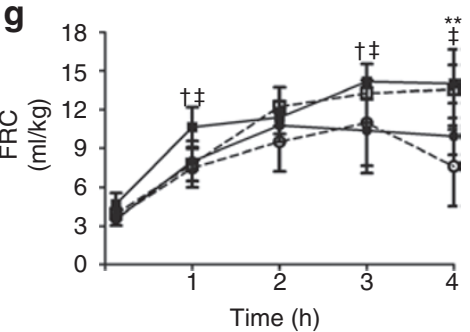

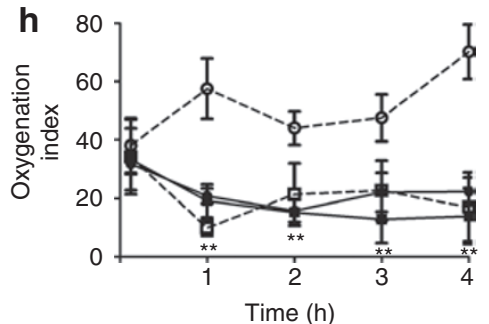

i

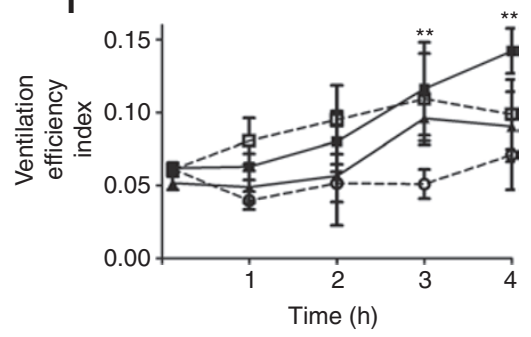

Figure 1. Lung physiological profile (mean \pm SD) in ventilated preterm lambs without surfactant treatment (negative controls, open circles), or SRT with beractant (filled circles), poractant alfa (open squares), or lucinactant (filled squares). (a) $\mathrm{PaO}_{2}$ increased as compared to baseline values and negative controls, independent of SRT group $\left({ }^{* *} P<0.001 ;{ }^{\dagger} P<0.05\right)$. Over time, the oxygenation response in lambs treated with lucinactant was sustained and greater than in lambs treated with beractant $(* P<0.001)$ or poractant alfa $\left({ }^{\ddagger} P<0.05\right)$. (b) $\mathrm{PaCO}_{2}$ decreased $(P<0.001)$ over time in all groups. Following $\mathrm{SRT}, \mathrm{PaCO}_{2}$ was lower than in negative controls for all SRT groups out to $2 \mathrm{~h}\left({ }^{*} P<0.001\right)$, beractant to $3 \mathrm{~h}\left({ }^{* *} P<0.01\right)$, and poractant alfa to $4 \mathrm{~h}\left({ }^{+} P<0.05\right)$ $(\ddagger P<0.05$ beractant vs. poractant alfa). (c) pH increased $(* * P<0.001)$ over time in all SRT groups and was greater than in negative controls, independent of SRT group. (d) Respiratory compliance increased $\left({ }^{*} P<0.001\right)$ as compared with baseline values and over time, independent of group. Improvement in compliance was greater $(* * P<001)$ in all SRT groups as compared with negative controls; no significant differences were noted between SRT groups. (e) PIP was significantly $(P<0.001)$ different over time as a function of group, lower in lucinactant-treated animals as compared with negative controls and animals treated with beractant at all time points $\left({ }^{* *} P<0.001 ;{ }^{\dagger} P<0.05\right)$ and poractant alfa $\left({ }^{\ddagger} P<0.05\right)$ at 3 to $4 \mathrm{~h}$. (f) PEEP increased $(P<0.001)$ over time, independent of group and was lower in lucinactant-treated animals at 3 to $4 \mathrm{~h}$ than in all other groups $\left({ }^{\ddagger} P<0.05 ;{ }^{* *} P<0.001\right)$. (g) FRC increased $(P<0.001)$ over time, independent of group and was greater in lucinactant- $\left({ }^{* *} P<0.001 ;^{\dagger} P<0.05\right)$ or poractant alfa-treated $\left({ }^{\ddagger} P<0.05\right)$ animals at 3 to $4 \mathrm{~h}$ than in those treated with beractant or negative controls. (h) Oxygenation index in all SRT animals was lower $(* * P<0.001)$ than baseline and negative controls; no significant differences between SRT groups were noted. (i) Ventilation efficiency index increased $(P<0.01)$ over time in all SRT animals and was greater ( $\left.{ }^{* *} P<0.001\right)$ in animals treated with lucinactant than in those treated with beractant at $3 \mathrm{~h}$ and all other groups at $4 \mathrm{~h}$. FRC, functional residual capacity; PEEP, positive end-expiratory pressure; PIP, peak inspiratory pressure; SRT, surfactant replacement therapy. 
independent of SRT group. Respiratory compliance (Figure 1d) increased $(P<0.001)$ as compared with baseline values and over time, independent of group. The improvement in compliance was greater $(P<0.001)$ in all SRT groups as compared with negative controls; no significant differences were noted between SRT groups. Peak inspiratory pressure (PIP, Figure 1e) was significantly $(P<0.001)$ different over time as a function of group. In comparison with baseline, PIP was greater $(P<0.001)$ in negative controls at all time points and lower in lucinactanttreated animals at 3 to $4 \mathrm{~h}$. Between SRT groups, PIP was lower $(P<0.001)$ in lucinactant-treated animals than those treated with beractant at all time points following treatment and those treated with poractant alfa at 3 to $4 \mathrm{~h}$. Positive end-expiratory pressure (Figure 1f) increased $(P<0.001)$ over time independent of group and was lower $(P<0.05)$ in lucinactant-treated animals at 3 to $4 \mathrm{~h}$ as compared with all other groups. Functional residual capacity (FRC, Figure 1g) increased $(P<0.001)$ over time independent of group and was greater $(P<0.05)$ in lucinactant- or poractant alfa-treated animals at 3 to $4 \mathrm{~h}$ as compared with negative controls or animals treated with beractant. The oxygenation index (Figure $1 \mathrm{~h}$ ) in all SRT animals was lower $(P<0.001)$ than in baseline and negative controls; no significant differences between SRT groups were noted. The ventilation efficiency index (Figure 1i) increased $(P<0.01)$ over time in all SRT groups and was greater $(P<0.001)$ in animals treated with lucinactant as compared with animals treated with beractant at $3 \mathrm{~h}$, and as compared with all groups at $4 \mathrm{~h}$.

\section{Lung Histological Profile}

Representative micrographs of matched regional lung sections obtained from a preterm lamb of each group are presented in Figure 2. Qualitatively, lungs treated with lucinactant appeared more homogenously expanded, with less cellular debris and fewer inflammatory cells as compared with lungs from all other groups. Quantitative histomorphological analysis (Figure 3) demonstrated that, independent of lung region, the expansion index (Figure $3 \mathrm{a}$ and $3 \mathrm{~b}$ ) of lungs from animals treated with SRT was greater $(P<0.01)$ than that of those not treated with surfactant. Regional differences in expansion were noted for beractant-treated lungs, with less $(P<0.001)$ expansion in the dependent than the nondependent region and as compared with the dependent region of lungs treated with lucinactant. The number of expanded gas exchange units (Figure $3 \mathrm{c}$ and 3d) was greater $(P<0.01)$ in lungs treated with lucinactant or poractant alfa than in those not treated with surfactant, independent of region. In comparison to beractant-treated lungs, the number of expanded gas exchange units was greater $(P<0.001)$ in those treated with lucinactant.

\section{Inflammatory Profile}

The lung (Figure 4) and systemic (Figure 5) inflammatory mediator profile overall was attenuated to a greater degree in lambs treated with lucinactant as compared with lambs in all other groups. Lung tissue levels of the proinflammatory mediators (Figure 4) interleukin (IL)-8, IL-6, and myeloperoxidase (MPO), independent of lung region, were lower $(p<0.01)$ following lucinactant treatment as compared with no surfactant treatment. Lung IL-8 (Figure 4a and 4b) following treatment with lucinactant was also lower as compared with beractant treatment $(P<0.01)$, and lung IL-8 was also lower following poractant alfa treatment as compared with negative controls $(P<0.05)$, independent of region. Lung IL-6 (Figure $4 \mathrm{c}$ and 4d) following lucinactant treatment was lower $(P<0.01)$ than in all other groups, independent of region. Lung MPO (Figure $4 \mathrm{e}$ and $4 \mathrm{f})$ was lower $(P<0.01)$ following lucinactant or beractant treatment as compared with negative controls, independent of region. Plasma IL-8 (Figure 5a) following lucinactant or poractant alfa treatment was lower $(P<0.01)$ as compared with negative controls; plasma IL-8 was also lower in lucinactant-treated animals as compared with animals treated with beractant $(P<0.01)$. Plasma IL-6 was lower $(P<0.01)$ in all SRT groups as compared with negative controls. In addition,
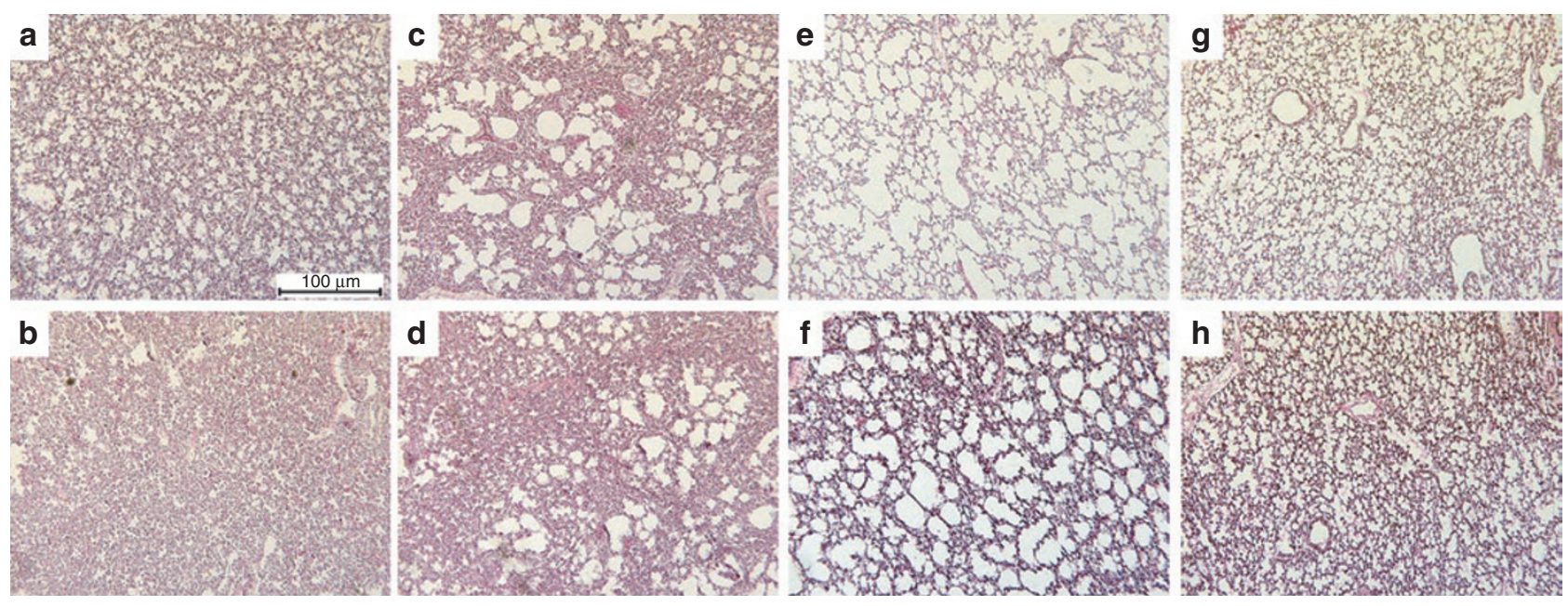

Figure 2. Representative micrographs of regional lung sections of matched area obtained from one preterm lamb of each group.

Top panels: nondependent lung; bottom panels: dependent lung. (a,b) Negative controls. (c,d) Beractant-treated. (e,f) Poractant alfa-treated.

(g,h) Lucinactant-treated. Original magnification: 100×. 
a
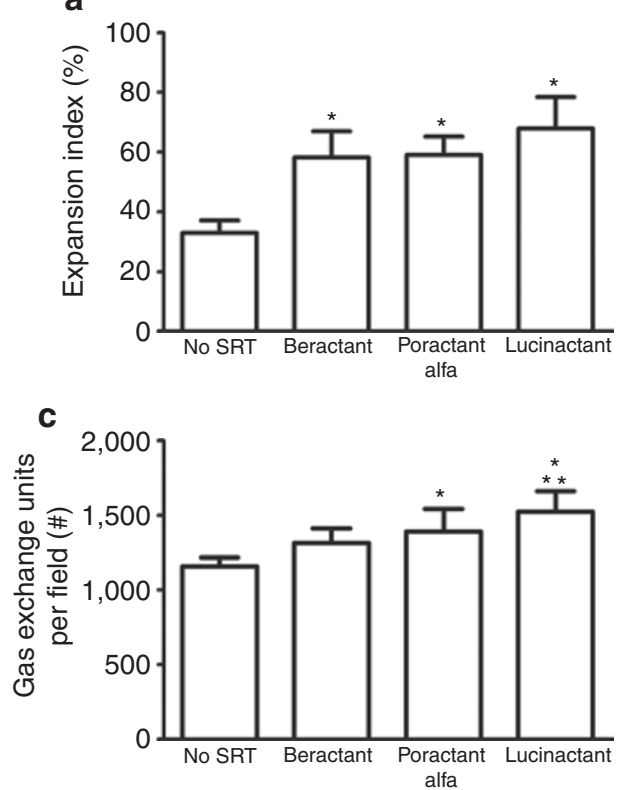

b
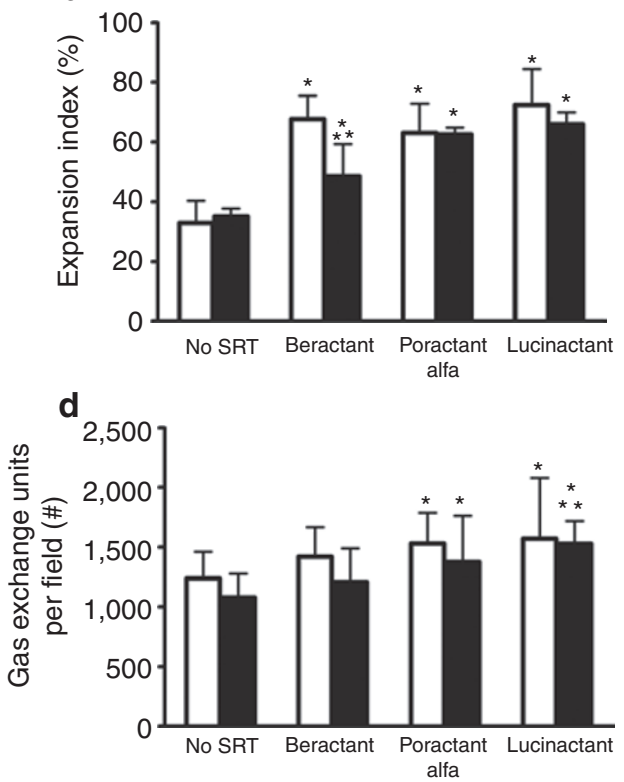

Figure 3. Histomorphological profile. (a) Expansion index, total lung. (b) Expansion index, regional lung. (c) Number of opened gas exchange units per fixed field, total lung. (d) Number of opened gas exchange units per fixed field, regional lung. For (b) and (d), open bars are nondependent, solid bars are dependent lung. Independent of lung region, the expansion index of lungs from lambs treated with SRT was greater $\left({ }^{*} P<0.01\right)$ than those not treated with surfactant. Regional differences in expansion were noted for beractant-treated lungs, with less $(* * P<0.001)$ expansion in the dependent than the nondependent region and as compared with the dependent region of lungs treated with lucinactant. The number of expanded gas exchange units was greater $\left({ }^{*} P<0.01\right)$ in lucinactant- or poractant alfa-treated lungs than those not treated with surfactant, independent of region. Relative to beractant-treated lungs, the number of expanded gas exchange units was greater $(* * P<0.001)$ in those treated with lucinactant. SRT, surfactant replacement therapy.

plasma IL-6 (Figure 5b) in lambs treated with either lucinactant or poractant alfa was lower $(P<0.05)$ as compared with animals treated with beractant.

\section{DISCUSSION}

SRT was first introduced more than $30 \mathrm{y}$ ago and is now considered standard of care for preterm infants with RDS. The goal of SRT in the preterm infant is to stabilize the alveolar space, thereby providing acute improvement in pulmonary compliance and lung volume, thus reducing the need, intensity, and duration of mechanical ventilation of the infant. SRT also contributes to the phospholipid substrate for endogenous production of surfactant. Of the four well-established surfactant proteins, SP-B has been identified as a key component of pulmonary surfactant's ability to reduce alveolar surface tension, prevent alveolar collapse and alveolar capillary narrowing, resist surfactant degradation, and protect against oxygen-induced lung injury $(16,18-20)$. Of note, SP-B is critical for viability; infants with SP-B deficiency die of respiratory failure after birth $(21,22)$.

In this study, preterm lambs treated with SRT predictably demonstrated improvements in oxygenation and lung mechanics that were higher as compared with lambs that did not receive SRT. Although differences in $\mathrm{PaCO}_{2}$ and $\mathrm{pH}$ were minimal between SRT groups, most notably, the improvement in oxygenation was sustained to a greater degree and ventilatory pressure requirements were lower in lambs treated with lucinactant. However, similar compliance improvements were observed in all SRT groups. The sustained improvement in oxygenation following lucinactant treatment is suggestive of a sustained reduction in intrapulmonary shunt that appears to be independent of the impact of improved lung mechanics. Previous studies have demonstrated that surfactants containing SP-B support alveolar capillary shape and pulmonary blood flow (19). In addition, surfactants that contain $\mathrm{KL}_{4}$ have been noted to decrease lung vascular permeability (23). These distinct physiological mechanisms provide a potential explanation for the uncoupling between sustained oxygenation seen only in animals treated with lucinactant and comparable improvements in lung mechanics observed in all animals receiving SRT.

Lungs treated with lucinactant were more homogeneously expanded within and between lung regions, with less cellular debris and fewer inflammatory cells noted as compared with all other groups. Uniformity of surfactant distribution has been shown to improve as the volume of surfactant administered is increased (24). The improvement in expansion pattern in our study is suggestive of more uniform distribution of the surfactant that is likely reflective of the relatively higher dosing volume of lucinactant as compared with both animalderived surfactants (25). Nonuniform distribution of surfactant and alveolar expansion predisposes to higher ventilatory requirements, volume and/or pressure induced lung injury in the overinflated alveoli, reduced surface area for gas exchange in the collapsed alveoli, structural disruption, and inflammatory cell infiltrate (26). Within this context, the improved 



Figure 4. Lung inflammatory profile. Total and regional lung tissue levels of (a,b) IL-8; (c,d) IL-6; (e,f) MPO. For (b), (d), and (f), open bars are nondependent, solid bars are dependent lung. Independent of lung region, all were lower $\left({ }^{*} P<0.01\right)$ following lucinactant treatment as compared with no surfactant treatment. Lung IL-8 following lucinactant treatment was also lower $\left({ }^{* *} P<0.01\right)$ than following treatment with beractant, and those treated with poractant alfa also had lower lung IL-8 $(* P<0.05)$ than did negative controls, independent of region. Lung IL-6 following lucinactant treatment was lower $(* P<0.01)$ than in all other groups, independent of region. Lung MPO was lower $(* P<0.01)$ following lucinactant or beractant treatment as compared with negative controls, independent of region. IL, interleukin; MPO, myeloperoxidase.

histological profile of lucinactant-treated lungs is consistent with the observation of lower ventilatory pressure requirements, thereby potentially reducing risk of lung injury and inflammation, while supporting ventilation and perfusion matching and resultant sustained oxygenation.

The qualitative appearance of less cellular debris and fewer inflammatory cells in lucinactant-treated lungs was substantiated by evaluating the tissue inflammatory profile. Lung proinflammatory cytokine markers were lower in preterm lambs treated with lucinactant than in both beractant-treated and negative control preterm lambs. Because such direct measurements are not possible in the preterm human, the context of these findings can be evaluated relative to previous in vitro or in vivo animal studies. A previous study demonstrated that lucinactant was more resistant than animal-derived surfactants to inhibition by both oxidants released during an inflammatory process and plasma proteins (27). In this regard, resistance to inhibition would preserve biological activity of the surfactant and support the attenuated lung inflammatory profile in the lucinactant-treated as compared with animal-derived surfactant-treated preterm lambs in our study. Our findings are also consistent with previously reported results that $\mathrm{KL}_{4}$ surfactant treatment resulted in lower levels of inflammatory markers in in vitro cell culture and in vivo adult models of hyperoxia-induced lung injury $(17,23)$. The attenuated inflammatory profile in our study is in contrast to a previous study in older preterm lambs that demonstrated no distinguishable differences in lung inflammation profile following treatment with natural sheep surfactant containing SP-A, SP-B, and SP-C; an organic solvent extract of natural sheep surfactant that contains SP-B and SP-C; beractant; or a synthetic surfactant containing recombinant SP-C and synthetic lipids (28). Although the difference in findings between studies may be related to differences in gestational age, other potential reasons for the attenuated inflammatory profile following treatment with lucinactant as compared with animal-derived surfactants in our study bears further discussion as noted below.

Relative to other hydrophobic surfactant proteins, SP-B plays a critical role in surfactant activity. Transgenic mice lacking SP-B and human infants with a genetic deficiency of SP-B experience severe fatal respiratory failure $(18,20-22,29)$; absence of SP-B blocks processing of SP-C (30). These studies 

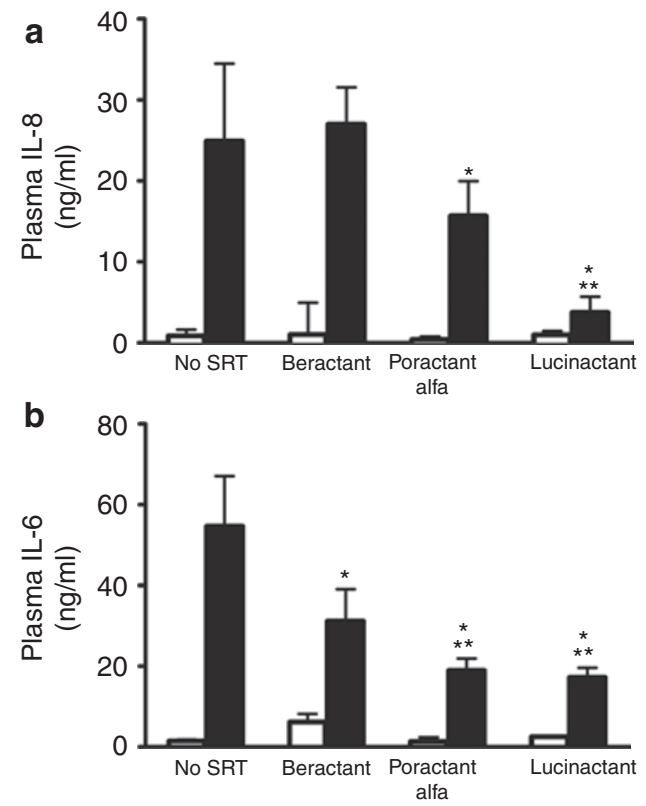

Figure 5. Plasma cytokine concentrations. Open bars are baseline and solid bars are $4 \mathrm{~h}$ values. (a). Plasma IL-8 following lucinactant or poractant alfa treatment was lower $\left({ }^{*} P<0.01\right)$ than in negative controls and those treated with lucinactant had lower plasma IL-8 levels than those treated with beractant $\left({ }^{* *} P<0.01\right)$. (b) Plasma IL-6 was lower $\left({ }^{*} P<0.01\right)$ in all SRT groups as compared with in negative controls. In addition, plasma IL-6 (b) in those treated with either lucinactant or poractant alfa was lower $\left({ }^{* *} P<0.05\right)$ than in those treated with beractant. IL, interleukin; SRT, surfactant replacement therapy.

indicate that the quantity of SP-B in a surfactant formulation impacts its function and other surfactant proteins. The amount of the SP-B mimetic, $\mathrm{KL}_{4}$, in lucinactant is consistently $2.9 \%$ of the total phospholipids, which is more than $3 \times$ greater than the amount of SP-B in beractant or poractant alfa (31-34). Within this context, the greater concentration and consistency of the SP-B mimetic, $\mathrm{KL}_{4}$, would favor greater protection against inflammation observed following lucinactant treatment relative to the animal-derived surfactants tested.

The role of lucinactant in attenuating inflammation may also be related to its interaction with cell membranes. Biophysical studies of $\mathrm{KL}_{4}$ demonstrating its ability to differentially partition into lipid lamellae and alter curvature strain suggest a mechanism for $\mathrm{KL}_{4}$ peptide-mediated lipid organization and trafficking within the dynamic lung environment $(35,36)$. By associating with lipid membranes, $\mathrm{KL}_{4}$ may cause cytoskeletal changes that could alter cell signaling potentially through inducing leucine zipper-related mechanisms of inhibiting AP-1 or NF-kB regulatory signaling pathways. The lower levels of IL-6 and IL-8 in lung homogenate from lucinactant-treated lungs of the preterm lamb are consistent with this proposed mechanism.

Palmitoyl-oleoyl-phosphatidylglycerol (POPG), a phospholipid in endogenous surfactant, has recently been shown to suppress the inflammatory response to respiratory syncytial virus and of macrophages and bronchial epithelial cells (i.e., IL- 6 and IL-8) elicited by ligands and activation of Toll-like receptors 2 and 3 (37). These findings raise the possibility that supplementation of the injured lung with POPG may suppress inflammation and hasten repair processes. Our results of attenuated lung inflammation as measured by lower levels of IL- 6 and IL-8 are consistent with these findings. To our knowledge, lucinactant is the only approved therapeutic surfactant with POPG identified as an active pharmaceutical ingredient. Within this context, it is plausible that POPG may be synergistically conveying an immunomodulatory effect through a mechanism separate and distinct from any $\mathrm{KL}_{4} /$ SP-B relationship.

Taken together, the results of this study suggest that lucinactant, and perhaps the $\mathrm{KL}_{4}$ peptide itself, modulates the inflammatory response in the lung. In addition, we noted lower circulating levels of IL- 6 and IL-8 following lucinactant treatment as compared with negative controls as well as both animal-derived surfactants, which in concert with the sustained improvement in oxygenation, reduced pressure requirements, and improved histomorphological profile relative to the other groups suggests that lucinactant is likely to afford protection against loss of compartmentalization leading to systemic inflammation.

The role of synthetic surfactants in SRT for management of RDS is highly relevant. At the most fundamental level, these compounds project fewer potential biohazardous effects than animal lung extracts. They do not contain immunologically incompatible contaminants, are not limited by livestock availability, are highly reproducible as compared with variability of animal-derived surfactants, and are less susceptible to inactivation by native proteases $(38,39)$. Clinical trials in preterm infants demonstrated significant benefits of lucinactant versus colfosceril palmitate (40), beractant (41), and poractant alfa (42), including fewer days receiving mechanical ventilation and days in the NICU (43), and reduced rates of reintubation (43), RDS-related mortality (40), and long term mortality (41), and improved survival without BPD (40). Because direct measurements of lung inflammatory mediators or structural outcomes were not part of the original study design of these SRT trials in human neonates, it is not known whether the outcomes could be related to differential SRT effects on lung inflammation. The results of our study are supportive of the role of lucinactant in attenuating lung inflammation and supporting lung structure as a contributing factor to the improved outcomes demonstrated in the clinical studies.

There are several experimental design considerations that warrant mention. This study was designed to evaluate physiologic, inflammatory, and lung structural responses to animal-derived and a synthetic surfactant delivered per clinical protocols and according to manufacturers' labels for dosing. As such, we cannot exclude differences in dosing volumes or phospholipid content as possible explanations for our results. Furthermore, given that our goal was to characterize the aforementioned profile in each animal, we chose not to label the surfactants and therefore cannot comment on the effect of differences in surfactant distribution. Finally, given that our study was only $4 \mathrm{~h}$ long, although we believe that an attenuated inflammatory profile in the lung may attenuate progression of RDS to BPD, studies of longer duration are required. 
In summary, the findings suggest that SRT with lucinactant incorporates mechanoprotective and cytoprotective mechanisms to attenuate lung and systemic inflammatory responses that are predictive of poor outcome in infants with RDS. Lucinactant attenuated lung inflammation and preserved lung structural integrity in preterm lambs with RDS to a greater degree than either no SRT or SRT with poractant alfa or beractant. These data suggest that early intervention with lucinactant may mitigate progression of pulmonary pathophysiological consequences of RDS.

\section{METHODS}

\section{Animal Protocol}

The experimental protocol and procedures were approved by the institutional animal care and use committee at Temple University School of Medicine and performed in accordance with National Institutes of Health guidelines.

Following cesarean section of the ewe, 24 fetal lambs (gestational age: $125-128 \mathrm{~d}$ ) were anesthetized (i.m.: $1 \mu \mathrm{g}$ Fentanyl; subcutaneous ventral neck: $\sim 1 \mathrm{ml} 0.5 \%$ lidocaine $\mathrm{HCl}$ ) and instrumented with an endotracheal tube by tracheotomy (3-3.5 mm ID Hi-Lo Jet Tube; Mallincrodkt, Saint Louis, MO) and carotid and venous catheters, paralyzed (i.v.: $0.10 \mathrm{mg} / \mathrm{kg}$ pancuronium bromide), and dried. After the umbilical cord was cut, the premature lamb was weighed, and time-cycled, pressure-limited ventilation (Infant Star Neonatal Ventilator, Infrasonics, San Diego, CA) was immediately initiated to target $\mathrm{PaCO}_{2}$ within $40-60 \mathrm{~mm} \mathrm{Hg}$, with ventilatory pressures and rate adjusted within the following settings to optimize pressure-volume relationships and to maintain physiological gas exchange and acid-base balance: $\mathrm{PIP} \leq 35 \mathrm{cmH}_{2} \mathrm{O}$, positive endexpiratory pressure $5-8 \mathrm{cmH}_{2} \mathrm{O}$, flow rate $\leq 10 \mathrm{l} / \mathrm{min}^{2}$ to provide a tidal volume $=4-6 \mathrm{ml} / \mathrm{kg}$, respiratory frequency $\leq 70$ breaths $/ \mathrm{min}$, with inspiratory time between $0.3-0.5 \mathrm{~s}$, and fraction of inspired oxygen $=1.0$. Airway temperature $\left(35^{\circ} \mathrm{C}\right)$ and humidity $(100 \%)$ were kept constant throughout the $4 \mathrm{~h}$ protocol.

Baseline measurements of arterial blood chemistry (Nova Statprofile M, Waltham, MA; Radiometer OSM 3, Copenhagen, Denmark), tidal volume by integrated pneumotachography signals (no. 00; Fleish, Eplinges, Switzerland), airway manometry, calculated respiratory compliance (PeDS-LAB, MAS, Hatfield, PA), FRC by closedcircuit helium dilution (Panda, MAS), mean arterial pressure, and heart rate were performed. Following baseline measurements, animals were randomly assigned to groups ( $n=6 /$ group): negative control (no surfactant), lucinactant (Surfaxin, Discovery Laboratories, Warrington, PA; $175 \mathrm{mg}$ total phospholipid/kg; $0.862 \mathrm{mg} / \mathrm{ml} \mathrm{KL}_{4}$; $5.8 \mathrm{ml} / \mathrm{kg}$ ), beractant (Survanta, Abbott Laboratories, Columbus, $\mathrm{OH} ; 100 \mathrm{mg}$ total phospholipid $/ \mathrm{kg} ; 0.10 \mathrm{mg} / \mathrm{ml} \mathrm{SP-B;} 4 \mathrm{ml} / \mathrm{kg}$ ), or poractant alfa (Curosurf, Chiesi Farmaceutici, S.p.A., Parma, Italy; $200 \mathrm{mg}$ total phospholipid/kg; $0.45 \mathrm{mg} / \mathrm{ml} \mathrm{SP-B;} 2.5 \mathrm{ml} / \mathrm{kg}$ ). Dose was administered consistent with manufacturers' prescribing information. Bolus instillation of the surfactant occurred over 5-10 min into the endotracheal tube through the wye of the ventilator circuit, during which time the animals were repositioned to optimize distribution using four equal aliquots of the total dose in Trendelenberg, reverse Trendelenberg, and left and right lateral decubitus positions.

Arterial pressure, heart rate, pulse oximetry, and rectal temperature were monitored continuously. Arterial blood chemistry and pulmonary mechanics were analyzed every $30 \mathrm{~min}$; FRC was measured and additional arterial blood samples were collected hourly for subsequent analyses of inflammatory mediators. Fluid and temperature management included $5 \%$ dextrose at $5 \mathrm{ml} / \mathrm{kg} / \mathrm{h}$, pancuronium bromide at $0.10 \mathrm{ml} / \mathrm{kg} / \mathrm{h}$ for paralysis to prevent confounding artifact from ineffectual respiratory efforts, buffer to correct $\mathrm{pH}$ (bicarbonate or nonbicarbonate based [THAM] on $\mathrm{pH}$ and $\mathrm{PCO}_{2}$ ), pressors (dopamine $\leq 20 \mu \mathrm{g} / \mathrm{kg} / \mathrm{min}$, if mean arterial pressure $\leq 40 \mathrm{~mm} \mathrm{Hg}$ ), radiant warming, and supplemental anesthesia as needed (fentanyl: $0.50 \mu \mathrm{g}$ if $20 \%$ increase in blood pressure following deep tissue stimulus). The oxygenation index and ventilatory efficiency index were computed as previously described (44).

\section{Lung Tissue Collection}

The animals were deeply anesthetized following the 4-h ventilation period. The trachea was clamped at the final end-expiratory pressure, the pressure correlated to support the FRC, and a midline thoracostomy with gross inspection was performed. The lungs were perfused through the vasculature with cold Millonig's phosphate buffer at $\leq 10$ $\mathrm{cmH}_{2} 0$ until the perfusate ran clear. The left and right lungs were prepared and dissected according to a predetermined matrix to support unbiased sampling procedures $(45,46)$. The left main stem bronchus and pulmonary artery were clamped, the left lung was removed and sectioned according to a predetermined matrix, yielding six consistent regional samples, with two samples each from the nondependent and dependent apex, mid, and base of the lung. Nondependent and dependent refer to the orientation of the lung region with respect to gravity where the dependent regions were dorsal. These samples were snap-frozen in liquid nitrogen and stored at $-70^{\circ} \mathrm{C}$ for subsequent analyses of proinflammatory mediators. The pulmonary vasculature of the right lung was perfused with buffered formalin at $\leq 10 \mathrm{cmH}_{2} 0$ and then the entire lung was stored in formalin, then dissected as described for the left lung, stored in formalin, washed in PBS, and stored in ethanol until prepared for standard paraffin embedding.

\section{Lung Histology and Inflammatory Biomarker Analysis}

Analyses were performed while blinded to treatment group. For histological analysis, embedded lung samples were step sectioned at $500 \mu \mathrm{m}$ intervals and a total of five, $5 \mu \mathrm{m}$ sections from each sample were slide-mounted and stained with hematoxylin and eosin. Lung sections were first viewed through a transparent grid matrix at low power and then changed to higher power to randomize selection. To eliminate sampling bias, every tenth grid region was then photographed and digitized. Morphometric image analysis (Image Pro Plus, Silver Spring, MD) was performed with customized algorithms that were free of geometric assumptions to assess the expansion index (ratio of volume of gas exchange to parenchymal space) by densitometry and to count the number of open gas exchange units per fixed field size using a modification of the radial alveolar count method $(45,47,48)$.

Cytokine protein concentration in lung tissue homogenate and plasma (IL-6 and IL-8) were determined by ovine-specific sandwich ELISA utilizing mouse anti-ovine capture antibody (IL-6 and IL-8: Serotec Raleigh, NC), rabbit anti-ovine detection antibody (IL-6 and IL-8; Serotec), and ovine cytokine protein as standard controls (IL-6 and IL-8; CSIRO, Clayton, South VIC, Australia) as previously described $(45,49)$. All standards and samples were run in duplicate. Standard curves were sensitive at $0.39-200 \mathrm{ng} / \mathrm{ml}$ for IL- 6 and $1.56-200 \mathrm{ng} / \mathrm{ml}$ for IL- 8 with correlation coefficients $>0.95$, and interassay and intra-assay coefficients of variance of $<10$ and $<5 \%$, respectively, for all assays.

Myeloperoxidase (MPO) concentration in lung tissue was determined with a previously described colorimetric bioassay $(49,50)$. Linear standard curves were obtained with sensitivity from 0.0625 to $1.44 \mathrm{U} / \mathrm{ml}$; interassay and intra-assay coefficients of variance were $<10$ and $<6 \%$, respectively.

Total IL-6, IL-8, and MPO concentrations in lung tissue were normalized to total protein concentration of lung homogenate. We focused on IL- 6 and IL- 8 and MPO as early markers of lung and systemic inflammation, and inflammatory cell infiltrate, respectively.

\section{Statistical Analysis}

Data and statistical analyses were performed using Microsoft Excel Groups and Graph Pad PRISM. Values are expressed as means \pm SD. For data that were normally distributed, the parametric ANOVA was used to establish a significant overall difference among the groups. If not normally distributed, the Kruskal-Wallis test was used for an overall comparison of group differences. Significance was accepted at $P<0.05$, and, when omnibus significance was found, Dunnett's post hoc comparison to the negative control was applied. Multiple pairwise comparisons among all groups were made with Dunn's test. 


\section{STATEMENT OF FINANCIAL SUPPORT}

This work was funded by a grant from Discovery Laboratories (to M.R.W., J.W., and T.H.S.); additional laboratory and personnel support was provided by grants from the Office of Naval Research (grants N00014-10-1-0928 and N0014-10-0761 to M.R.W.) and the National Institutes of Health (grant 1P20RR020173 to T.H.S.).

\section{REFERENCES}

1. Jobe AH, Ikegami M. Prevention of bronchopulmonary dysplasia. Curr Opin Pediatr 2001;13:124-9.

2. Lodha A, Asztalos E, Moore AM. Cytokine levels in neonatal necrotizing enterocolitis and long-term growth and neurodevelopment. Acta Paediatr 2010;99:338-43.

3. American Academy of Pediatrics - Committee on fetus and newborn and Canadian Paediatric Society - Fetus and newborn committee. Postnatal corticosteroids to treat or prevent chronic lung disease in preterm infants. Pediatrics 2002;109:330-8.

4. Sakurai R, Li Y, Torday JS, Rehan VK. Curcumin augments lung maturation, preventing neonatal lung injury by inhibiting TGF- $B$ signaling. Am J Physiol Lung Cell Mol Physiol 2011;301:L721-30.

5. George CL, Goss KL, Meyerholz DK, Lamb FS, Snyder JM. Surfactantassociated protein A provides critical immunoprotection in neonatal mice. Infect Immun 2008;76:380-90.

6. Sato A, Whitsett JA, Scheule RK, Ikegami M. Surfactant protein-d inhibits lung inflammation caused by ventilation in premature newborn lambs. Am J Respir Crit Care Med 2010;181:1098-105.

7. Hilgendorff A, Parai K, Ertsey R, et al. Inhibiting lung elastase activity enables lung growth in mechanically ventilated newborn mice. Am J Respir Crit Care Med 2011;184:537-46.

8. Wallace MJ, Probyn ME, Zahra VA, et al. Early biomarkers and potential mediators of ventilation-induced lung injury in very preterm lambs. Respir Res 2009;10:19.

9. Albertine KH, Jones GP, Starcher BC, et al. Chronic lung injury in preterm lambs. Disordered respiratory tract development. Am J Respir Crit Care Med 1999;159:945-58.

10. Coalson JJ, Winter VT, Siler-Khodr T, Yoder BA. Neonatal chronic lung disease in extremely immature baboons. Am J Respir Crit Care Med 1999;160:1333-46.

11. Groneck P, Speer CP. Inflammatory mediators and bronchopulmonary dysplasia. Arch Dis Child Fetal Neonatal Ed 1995;73:F1-3.

12. Bose CL, Dammann CE, Laughon MM. Bronchopulmonary dysplasia and inflammatory biomarkers in the premature neonate. Arch Dis Child Fetal Neonatal Ed 2008;93:F455-61.

13. van Kaam AH, Haitsma JJ, Dik WA, et al. Response to exogenous surfactant is different during open lung and conventional ventilation. Crit Care Med 2004;32:774-80.

14. Stamme C, Brasch F, von Bethmann A, Uhlig S. Effect of surfactant on ventilation-induced mediator release in isolated perfused mouse lungs. Pulm Pharmacol Ther 2002;15:455-61.

15. van Iwaarden JF, Claassen E, Jeurissen SH, Haagsman HP, Kraal G. Alveolar macrophages, surfactant lipids, and surfactant protein $\mathrm{B}$ regulate the induction of immune responses via the airways. Am J Respir Cell Mol Biol 2001;24:452-8.

16. Cochrane CG, Revak SD. Pulmonary surfactant protein B (SP-B): structure-function relationships. Science 1991;254:566-8.

17. Zhu Y, Miller TL, Chidekel A, Shaffer TH. KL4-surfactant (Lucinactant) protects human airway epithelium from hyperoxia. Pediatr Res 2008;64:154-8.

18. Tokieda K, Whitsett JA, Clark JC, et al. Pulmonary dysfunction in neonatal SP-B-deficient mice. Am J Physiol 1997;273(4 Pt 1):L875-82.

19. Ikegami M, Weaver TE, Grant SN, Whitsett JA. Pulmonary surfactant surface tension influences alveolar capillary shape and oxygenation. Am J Respir Cell Mol Biol 2009;41:433-9.

20. Whitsett JA, Weaver TE. Hydrophobic surfactant proteins in lung function and disease. N Engl J Med 2002;347:2141-8.

21. Nogee LM, de Mello DE, Dehner LP, Colten HR. Brief report: deficiency of pulmonary surfactant protein B in congenital alveolar proteinosis. N Engl J Med 1993;328:406-10.
22. Hamvas A, Cole FS, deMello DE, et al. Surfactant protein B deficiency: antenatal diagnosis and prospective treatment with surfactant replacement. J Pediatr 1994;125:356-61.

23. Kinniry P, Pick J, Stephens S, et al. KL4-surfactant prevents hyperoxic and LPS-induced lung injury in mice. Pediatr Pulmonol 2006;41:916-28.

24. Gilliard N, Richman PM, Merritt TA, Spragg RG. Effect of volume and dose on the pulmonary distribution of exogenous surfactant administered to normal rabbits or to rabbits with oleic acid lung injury. Am Rev Respir Dis 1990;141:743-7.

25. Terry MH, Merritt TA, Harding B, et al. Pulmonary distribution of lucinactant and poractant alfa and their peridosing hemodynamic effects in a preterm lamb model of respiratory distress syndrome. Pediatr Res 2010;68:193-8.

26. Lewis JF, Ikegami M, Jobe AH, Absolom D. Physiologic responses and distribution of aerosolized surfactant (Survanta) in a nonuniform pattern of lung injury. Am Rev Respir Dis 1993;147(6 Pt 1):1364-70.

27. Manalo E, Merritt TA, Kheiter A, Amirkhanian J, Cochrane C. Comparative effects of some serum components and proteolytic products of fibrinogen on surface tension-lowering abilities of beractant and a synthetic peptide containing surfactant KL4. Pediatr Res 1996;39:947-52.

28. Ikegami M, Jobe AH. Injury responses to different surfactants in ventilated premature lamb lungs. Pediatr Res 2002;51:689-95.

29. Clark JC, Wert SE, Bachurski CJ, et al. Targeted disruption of the surfactant protein B gene disrupts surfactant homeostasis, causing respiratory failure in newborn mice. Proc Natl Acad Sci USA 1995;92:7794-8.

30. Beers MF, Hamvas A, Moxley MA, et al. Pulmonary surfactant metabolism in infants lacking surfactant protein B. Am J Respir Cell Mol Biol 2000;22:380-91.

31. Cochrane CG, Revak SD, Merritt TA, et al. The efficacy and safety of KL4surfactant in preterm infants with respiratory distress syndrome. Am J Respir Crit Care Med 1996;153:404-10.

32. Wang Z, Baatz JE, Holm BA, Notter RH. Content-dependent activity of lung surfactant protein B in mixtures with lipids. Am J Physiol Lung Cell Mol Physiol 2002;283:L897-906.

33. Notter RH, Wang Z, Egan EA, Holm BA. Component-specific surface and physiological activity in bovine-derived lung surfactants. Chem Phys Lipids 2002;114:21-34.

34. van Eijk M, De Haas CG, Haagsman HP. Quantitative analysis of pulmonary surfactant proteins B and C. Anal Biochem 1995;232:231-7.

35. Antharam VC, Elliott DW, Mills FD, Farver RS, Sternin E, Long JR. Penetration depth of surfactant peptide KL4 into membranes is determined by fatty acid saturation. Biophys J 2009;96:4085-98.

36. Long JR, Mills FD, Ganesh OK, Antharam VC, Farver RS. Partitioning, dynamics, and orientation of lung surfactant peptide $\mathrm{KL}(4)$ in phospholipid bilayers. Biochim Biophys Acta 2010;1798:216-22.

37. Numata M, Chu HW, Dakhama A, Voelker DR. Pulmonary surfactant phosphatidylglycerol inhibits respiratory syncytial virus-induced inflammation and infection. Proc Natl Acad Sci USA 2010;107:320-5.

38. Merritt TA, Strayer DS, Hallman M, Spragg RD, Wozniak P. Immunologic consequences of exogenous surfactant administration. Semin Perinatol 1988;12:221-30.

39. Strayer DS, Hallman M, Merritt TA. Immunogenicity of surfactant. II. Porcine and bovine surfactants. Clin Exp Immunol 1991;83:41-6.

40. Moya FR, Gadzinowski J, Bancalari E, et al.; International Surfaxin Collaborative Study Group. A multicenter, randomized, masked, comparison trial of lucinactant, colfosceril palmitate, and beractant for the prevention of respiratory distress syndrome among very preterm infants. Pediatrics 2005;115:1018-29.

41. Moya F, Sinha S, Gadzinowski J, et al.; SELECT and STAR Study Investigators. One-year follow-up of very preterm infants who received lucinactant for prevention of respiratory distress syndrome: results from 2 multicenter randomized, controlled trials. Pediatrics 2007;119:e1361-70.

42. Sinha SK, Lacaze-Masmonteil T, Valls i Soler A, et al.; Surfaxin Therapy Against Respiratory Distress Syndrome Collaborative Group. A multicenter, randomized, controlled trial of lucinactant versus poractant alfa among very premature infants at high risk for respiratory distress syndrome. Pediatrics 2005;115:1030-8. 
43. Guardia CG, Moya FR, Sinha S, et al. Reintubation and risk of morbidity and mortality in preterm infants after surfactant replacement therapy. J Neonatal Perinatal Med 2011;4:101-9.

44. Notter RH, Egan EA, Kwong MS, Holm BA, Shapiro DL. Lung surfactant replacement in premature lambs with extracted lipids from bovine lung lavage: effects of dose, dispersion technique, and gestational age. Pediatr Res 1985;19:569-77.

45. Wolfson MR, Hirschl RB, Jackson JC, et al. Multicenter comparative study of conventional mechanical gas ventilation to tidal liquid ventilation in oleic acid injured sheep. ASAIO J 2008;54:256-69.

46. Hsia CC, Hyde DM, Ochs M, Weibel ER; ATS/ERS Joint Task Force on Quantitative Assessment of Lung Structure. An official research policy statement of the American Thoracic Society/European Respiratory Society: standards for quantitative assessment of lung structure. Am J Respir Crit Care Med 2010;181:394-418.
47. Wolfson MR, Greenspan JS, Deoras KS, Rubenstein SD, Shaffer TH. Comparison of gas and liquid ventilation: clinical, physiological, and histological correlates. J Appl Physiol 1992;72:1024-31.

48. Deoras KS, Wolfson MR, Searls RL, Hilfer SR, Sheffield JB, Shaffer TH. Use of a touch sensitive screen and computer assisted image analysis for quantitation of developmental changes in pulmonary structure. Pediatr Pulmonol 1990;9:109-18

49. Shashikant BN, Miller TL, Welch RW, Pilon AL, Shaffer TH, Wolfson MR. Dose response to rhCC10-augmented surfactant therapy in a lamb model of infant respiratory distress syndrome: physiological, inflammatory, and kinetic profiles. J Appl Physiol 2005;99: 2204-11.

50. Schierwagen C, Bylund-Fellenius AC, Lundberg C. Improved method for quantification of tissue PMN accumulation measured by myeloperoxidase activity. J Pharmacol Methods 1990;23:179-86. 